\title{
STUDY ON THE SAFETY MANAGEMENT OF TOXIC GAS CYLINDER DISTRIBUTION USING RFID
}

\author{
BO-HEE SONG., JIN-HAN LEE., YOUNG-DO JO \& KI-DONG PARK \\ Korea Gas Safety Corporation, South Korea.
}

\begin{abstract}
The usage of toxic gas has increased consistently by the needs of the high-tech industry. Unfortunately, the number of toxic gas accident has also increased and it might cause huge human and material damage which is occurred by toxic gas leakage and diffusion. Mainly, toxic gases are stored in cylinders, however, there is a high probability of abusing to terrorism for toxic gas cylinder and operators dealing with toxic gas manage their toxic gas cylinder individually. For these reasons, toxic gas cylinder needs tracking management on a national level. In this study, it suggests that the tracking management system of toxic gas cylinder using RFID (Radio-Frequency identification), which is able to handle systematically by data integrating with equal cylinder distribution in each company. It is essential that the data integration system of toxic gas cylinder must establish a standardized ID on each cylinder and manage the history of cylinder distribution on existing each system. This system is operating preliminarily on the three sites to verify. It is expected that accidents caused by terror will be dramatically reduced and the most advanced level of safety control will be achieved.
\end{abstract}

Keywords: cylinder, distribution, toxic gas, RFID, tracking system.

\section{INTRODUCTION}

The usage of toxic gas has increased consistently by the needs of the high-tech industry. Unfortunately, many accidents related to the toxic gas have been increasing in the industrial field. It might cause huge human and material damage which is occurred by toxic gas leakage and diffusion. Especially, toxic gas has a high probability of abusing to terrorism because it is stored in a cylinder and may be located near residential area generally. In some cases, the maintenance of these cylinders may be required by company rules individually. So, management and tracking of total toxic gas cylinders could be complicated and difficult task nationally because of non-standard in maintenance of gas cylinders. Therefore, systematic techniques are inevitable to construct the tracking and management systems for toxic gas cylinders on a national level.

In this study, it suggests that the tracking management system of toxic gas cylinder using RFID (Radio-Frequency identification). RFID technology uses electromagnetic fields to automatically identify and track the tags attached to the gas cylinder. The tags contain electronically stored information. Unlike a barcode, the tags don't need to be within the line of sight, and it is just embedded in the tracked cylinder. Also, the tags have a large storage capacity and recognition rate compared with barcode. This advantageous of RFID shows the very suitable method for tracking and management systems for toxic gas cylinders [2]. 


\section{SYSTEM OVERVIEW}

\subsection{Management system for toxic gas cylinder using RFID}

The hardware architecture of the system consists of an RFID tag, RFID tag reader, web server and database server. The web server and database server are located in the main station. The tag readers are distributed around gas supplier and end user. Communication between the tag reader and the main station is done via internet. The software architecture consists of a communication driver that handles all communication functions done at the main station, an analyser the safety of gas cylinder at a location with GIS information of buildings, a friendly GUI, a device to notify the safety manager via cell phone, and a database that saves all readings and the history of gas cylinders.

The information collected from the main station includes general cylinder information, distribution information, and inspection information. The cylinder information is including the cylinder identification number, owner (company), material information, weight, filing date and filling companies. The distribution information is including cylinder Delivery Company, arrival date, person who placed the order and cylinder information. The inspection information is including the cylinder inspection place and expiry date of inspection. In the case of cylinders to be delivered to overseas countries, the collecting information of main station is the country, delivery date, and recovery date.

The safety checks in the main station perform the following works. It may generate an alarm to the safety manager under abnormal situations, such as a cylinder may be delayed in a time more than a designated time ( $24 \mathrm{hr}$ ) for its purpose, or a cylinder's inspection date or withdraw date may be due. It checks also with provided guidelines on the recommended maximum capacity of toxic gas cylinder near schools, hospitals etc.

\subsection{Flow diagram for the safety check}

Figure 2 shows the flow chart of safety check in the main station for returned cylinder. The first step is tagging the returned cylinder automatically by readers and next is sending an identification number of cylinders to the main station, and then a judgement is made on whether the cylinder is empty or not. If toxic gas remains in the cylinder, the application

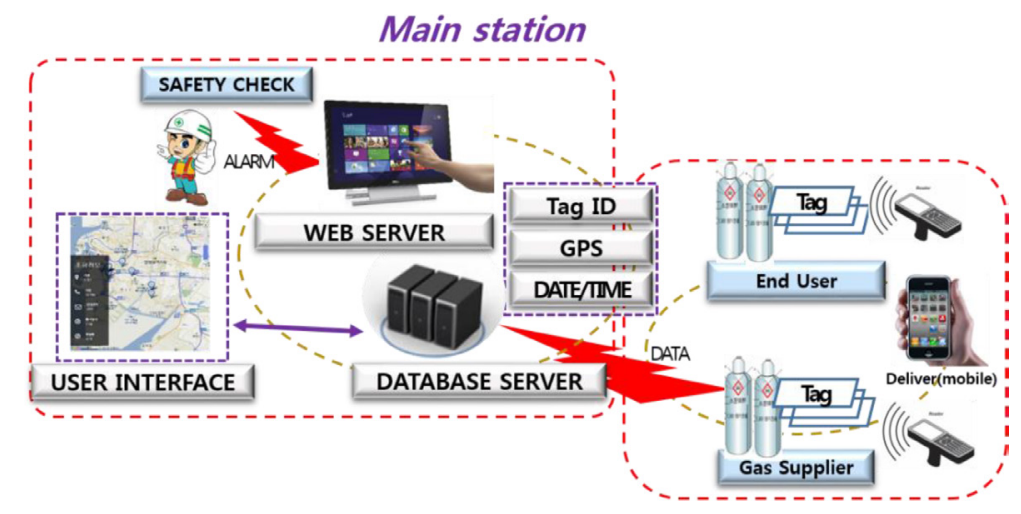

Figure 1: Management system for toxic gas cylinder. 


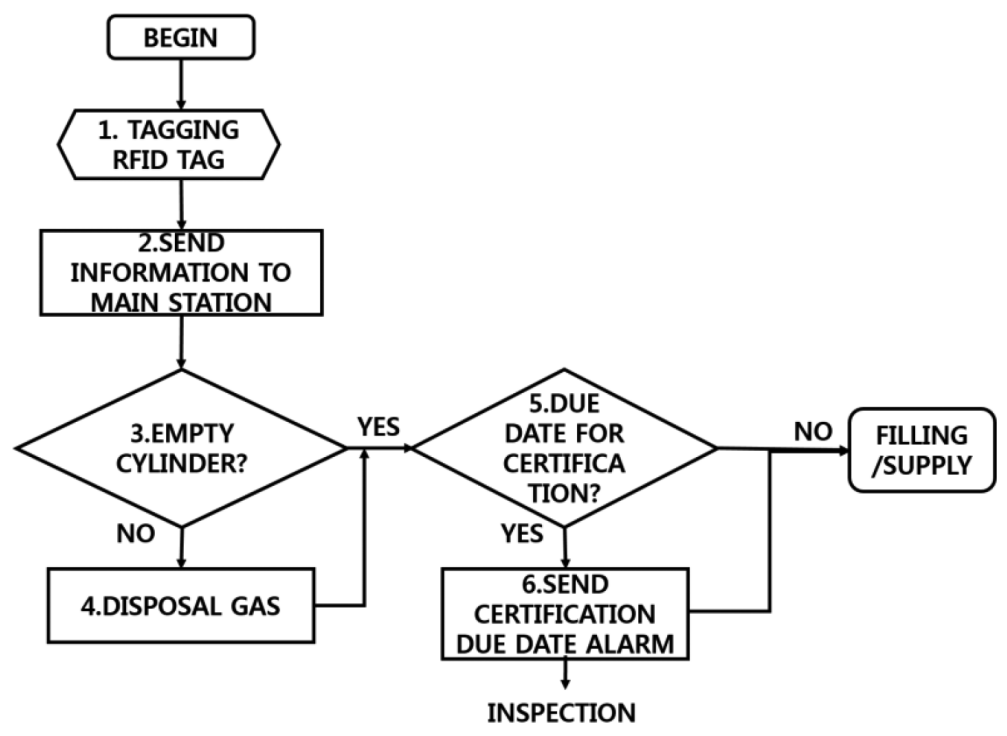

Figure 2: Safety check flow diagram for returned cylinder.

moves to step 4 where system orders to disperse the toxic gas in the cylinder at safe place. In step 5, checking is done on whether the gas cylinder is inspected or not. The toxic gas cylinder should be inspected regularly by regulation in Korea. If the inspection date is expected to come within a week, the system moves to step 5 to send an alarm of the inspection requirement to the safety manager. If it is confirmed that there is no problem with the above safety check, the cylinder is filled with toxic gas and supply to end user.

Figure 3 shows the flow chart of the sequence of application in the main station for delivering the cylinder filled with toxic gas. A judgement is made on whether the delivery time of the cylinder is appropriate or not in the 3rd step. If the delivery of gas cylinder is delayed at an inappropriate time, i.e. not within $24 \mathrm{hr}$, the application sends an alarm of delayed delivery of the cylinder to the safety manager. When the system received ID data of the cylinder from the site of the end user, the system checks vulnerable buildings, such as a school or hospital, in the hazard area, which is calculated based on the amount of toxic gas in the cylinder. If there is a vulnerable building in the hazard area, the system recommends some measures to the safety manager to reduce consequences, such as reduce the size of toxic gas cylinder.

Figure 4 shows the flow chart of the sequence of application in the main station for imported cylinder by overseas. Imported cylinder of toxic gas should be retrieved back by the supplier according to regulation in 6 months in Korea. A judgement is made on whether to retrieve the imported gas cylinder or not. If the due date of retrieve is expected to occur within a week, the system sends an alarm of retrieve to the safety manager.

\section{FIELD TEST}

Field tests of an RFID tracking system of gas cylinder were conducted to verify the function of the system by tagging and tracking to 60 chloride cylinders of UNID, 600 ammonia cylinders of Airtech, and 600 hydrogen chloride cylinders of Hong-In Chemical Company. These companies were selected because of small company not to do themselves. Before the tracking system application, it is essential to give a unique ID to each gas cylinder to safety manage 


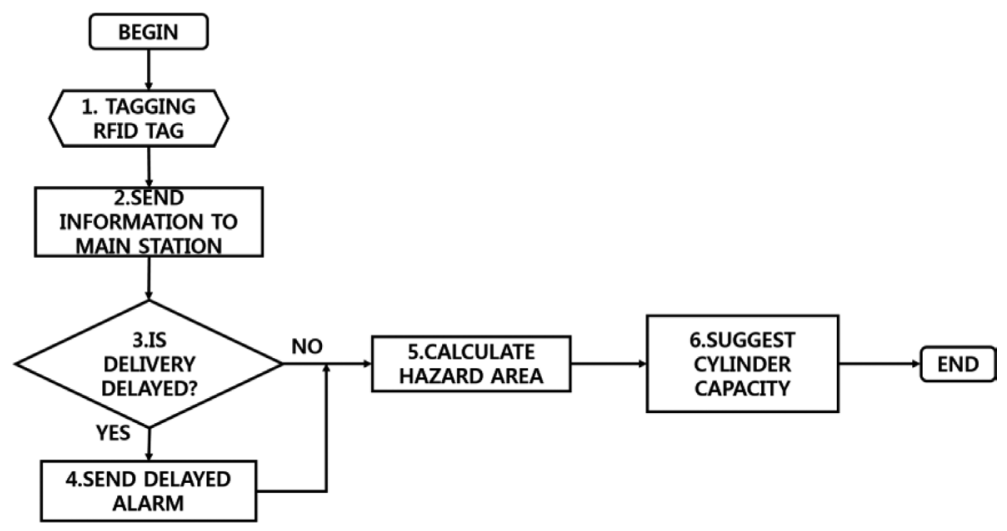

Figure 3: Safety check flow diagram for delivering the cylinder filled with toxic gas.

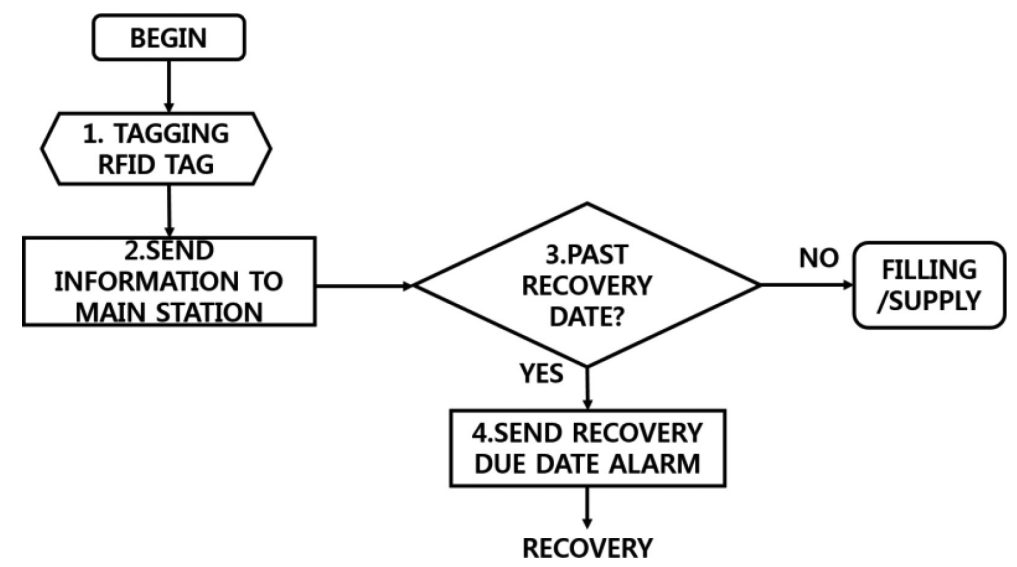

Figure 4: Safety check flow diagram for imported cylinder by overseas.

each gas cylinder. The ID of the tag to be attached to toxic gas cylinders was standardized as shown in Fig. 5.

The ID, standardized based on ISO 15459, consists of national code of Korea as KKR, institute code as CGS, material code of toxic gas for one space, company code for two spaces, and serial number for three spaces. In Korea, about 36 toxic gas materials are interested for high toxic gas and about 200 companies of toxic gas cylinder supplier are interested. Therefore, the above code can cover all toxic gas cylinders which should be tracked in Korea.

The type and attachment method of RFID tags differ depending on the type of gas cylinder selected and the field test has been underway since Sept. 2015.

1. Field test for chloride cylinders of UNID: 1-ton chloride welded cylinders were tested as shown in Fig. 6. If the RFID is attached to the surface of cylinder body, it may be damaged during handling the cylinder for a long time. Therefore, a ring type RFID tag is attached to the cylinder valve. The test was performed in the Incheon area.

2. Field test for ammonia cylinders of Airtech: The container of ammonia is a 47 L-ammonia welded cylinder. The RFID tag is attached to the top side of the cylinder where does not contact to other cylinder during treatment. This test was performed in the Daegu area. 


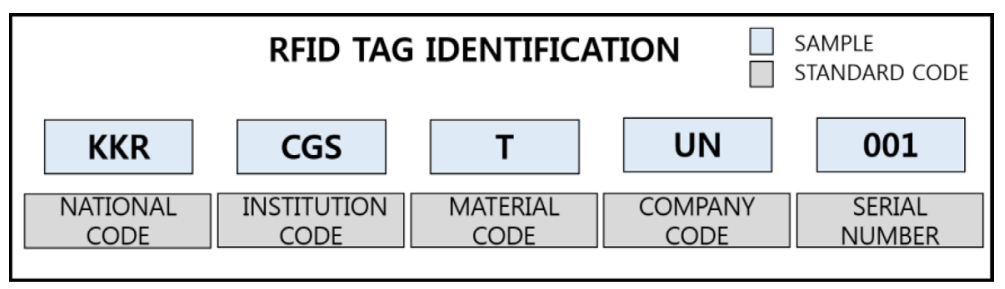

Figure 5: Standard code of RFID TAG and sample.
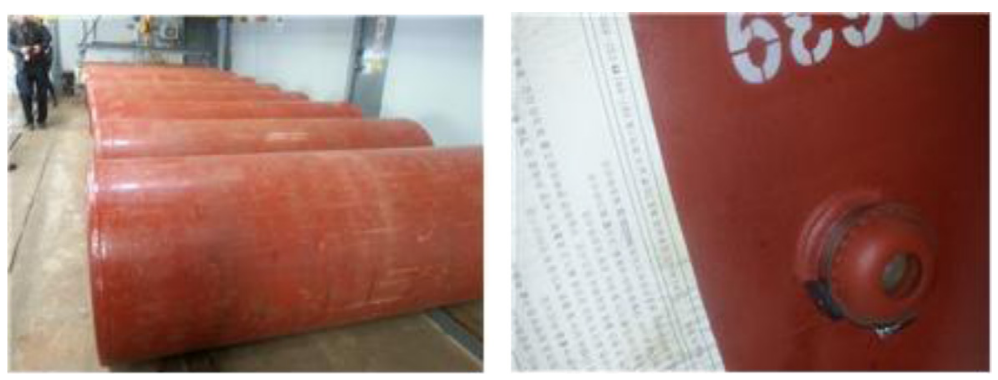

Figure 6: Field test of 1 tone chlorine cylinder.

3. Field test for hydrogen chloride cylinders of Hong-In Chemical: The hydrogen chloride is delivered by using $47 \mathrm{~L}$ chloride seamless cylinders. The tag was made in the manner of the Airtech cylinder tags, which was attached at the topside of the cylinder.

There is no difficult and no problem in the field test until now. The safety management with the tracking system is described in detail in the following sections as an example for chlorine.

\section{ANALYSIS OF THE EFFECTIVE DISTANCE OF TOXIC FOR CHLORINE}

The toxicity of chlorine gas depends on the amount of chlorine gas exposed to a person, the time of exposure, and pathway. Chlorine is a mild irritation to the mucus membranes during low exposure of 1-3 ppm, and at $30 \mathrm{ppm}$ immediate chest pain, shortness of breath, and a cough develops. The acute exposure guideline level-3, that person after exposing $1 \mathrm{hr}$ could experience life-threatening adverse health effects or death, is $20 \mathrm{ppm}$ of chlorine [3]. Based on the concentration level of chlorine gas, the effective distance of toxicity on person inside or outside the building is analysed.

The effective distance on person outside building for an instantaneous release of toxic gas at ground level is calculated conservatively by using the Gaussian puff model as the following [3].

$$
X_{\text {hazard }}=\left[\frac{a_{1} Q}{(2 \pi)^{3 / 2} C_{c r}}\right]^{\frac{1}{b_{1}}}
$$

Where $\mathrm{Q}$ is the total mass of released toxic gas, $C_{c r}$ is the lethal concentration of toxic gas as $20 \mathrm{ppm}$, and $\mathrm{a}_{1}$ and $\mathrm{b}_{1}$ are constants. The constants depend very sensitively on the atmospheric stability class. The effective distance may be defined as hazardous distance because a person being far away from the distance has no toxic effect. 

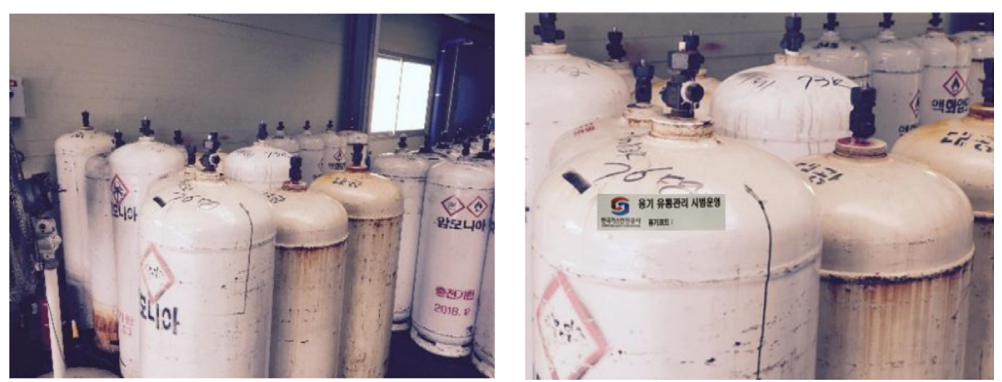

Figure 7: Field test of $47 \mathrm{~L}-$ ammonia cylinder.
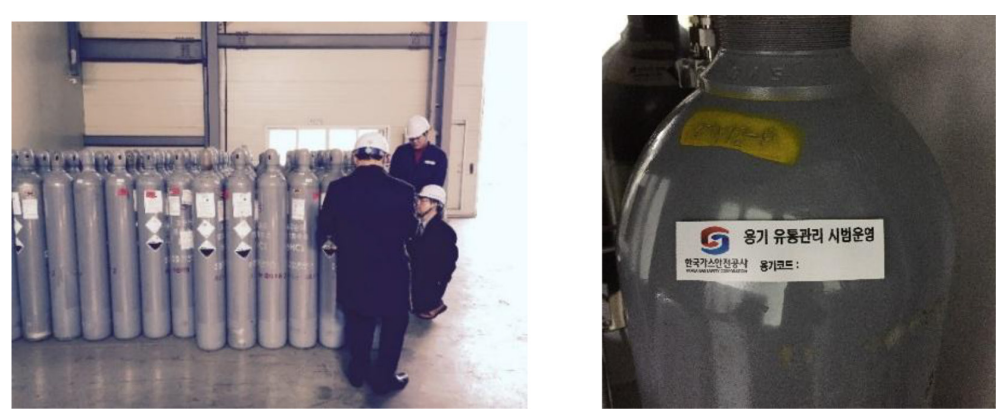

Figure 8: Field test of 47 L-hydrogen chloride cylinder.

The distance toxic effecting on person inside building in down-wind can be defined as critical distance because the person has toxic hazard without he/she's situation. The critical distance can be estimated approximately by using the following eqn (3).

$$
X_{c r}=\left[\frac{0.0007 a 2 Q}{(2 \pi)^{3 / 2} C_{c r}} \frac{\bar{\eta}}{U_{w}}\right]^{\frac{1}{b 2}}
$$

Where $\bar{\eta}$ is ventilation ratio and $U_{W}$ is wind velocity.

The constants in the eqns (1) and (2) can be obtained by using Pasquil-Gifford dispersion coefficients. The constants are summarized with the stability class in the Table 1 . The constant, a, is sesivively affected by the stability class which is in the range from 9.3 to 5,000 . The constant, $b$, is in the range from 1.5 to 2.6.

The hazard and critical distances are calculated by the total amount of chlorine gas released for class $\mathrm{C}$ by using the constants in Table 1, and results are shown in Fig. 9. The hazard distance from chlorine release may be up to several ten kilometres. It might be too conservative to use as a control parameter of the chlorine gas cylinder for approaching to vulnerable site, such as school or hospital. A person located from a critical distance to hazard distance might be safe in a building with sealing doors and windows with wet material and wait until the toxic plume is gone away. Therefore, the critical distance can be used reasonably as a safety control parameter. 
Table 1: The constants with Pasquil-Gifford stability class.

\begin{tabular}{lrlrrr}
\hline & \multicolumn{2}{c}{$X_{\text {hazard }}$} & & \multicolumn{2}{c}{$X_{\text {critica }}$} \\
\cline { 2 - 3 } \cline { 5 - 6 } Pasquill-Gifford & \multicolumn{1}{c}{$a_{1}$} & $b_{1}$ & & $a_{2}$ & $b_{2}$ \\
\hline A & 51 & 2.59 & 9.3 & 1.67 \\
B & 96 & 2.57 & 13.5 & 1.65 \\
C & 294 & 2.55 & 29.4 & 1.63 \\
D & 1,852 & 2.54 & 111.1 & 1.62 \\
E & 6,250 & 2.49 & 250 & 1.57 \\
F & 50,000 & 2.39 & 1,000 & 1.5 \\
\hline
\end{tabular}

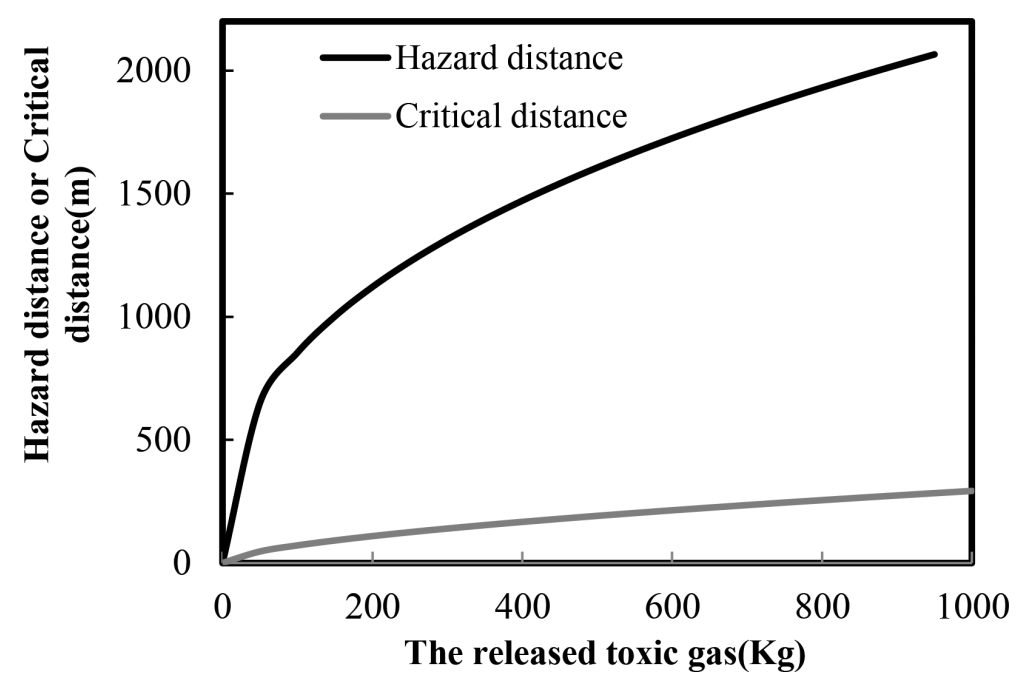

Figure 9: Comparing the hazard distance and critical distance for toxic gas release (stability class $\left.\mathrm{C}, \bar{\eta} / U_{W}=0.5, \mathrm{C}_{\mathrm{cr}}=20 \mathrm{ppm}\right)$.

The critical distance associated with the total amount of released chlorine gas ranges from under few tens meters for atmospheric stability class A (extremely unstable) and is up to over 1,000 meters for atmospheric stability class E (slightly stable) as shown in Fig. 10.

The critical distance also depends on the wind velocity and infiltration rate as shown in Fig. 11. If we assume the range of wind velocity from $1 / 7,200 \mathrm{~m} / \mathrm{sec}$ to $40 \mathrm{~m} / \mathrm{sec}$ and infiltration rate from $0.3 / \mathrm{hr}$ to $1 / \mathrm{hr}$, the worst accident scenario is that the ratio of infiltration rate to wind velocity $\left(\bar{\eta} / U_{W}\right)$ is 2 and the atmospheric stability is F class. The other extreme scenario is that the ratio is 0.003 and the stability is A. The critical distances for the two accident scenarios can be calculated by using eqn (2) and Table 1 .

For $\bar{\eta} / U_{W}=0.003$ and atmospheric stability class $=\mathrm{A}$,

$$
X_{1-c r} \cong 0.201 Q^{\frac{1}{1.67}}
$$




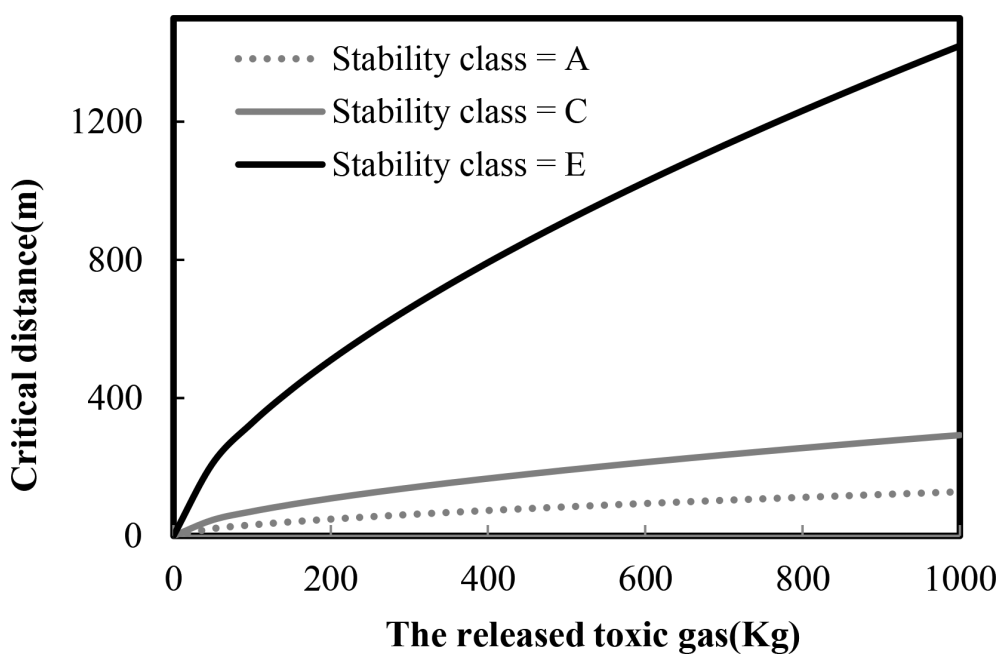

Figure 10: Effect of atmospheric stability on critical distances $\left(\bar{\eta} / U_{W}=0.5, \mathrm{C}=20 \mathrm{ppm}\right)$.

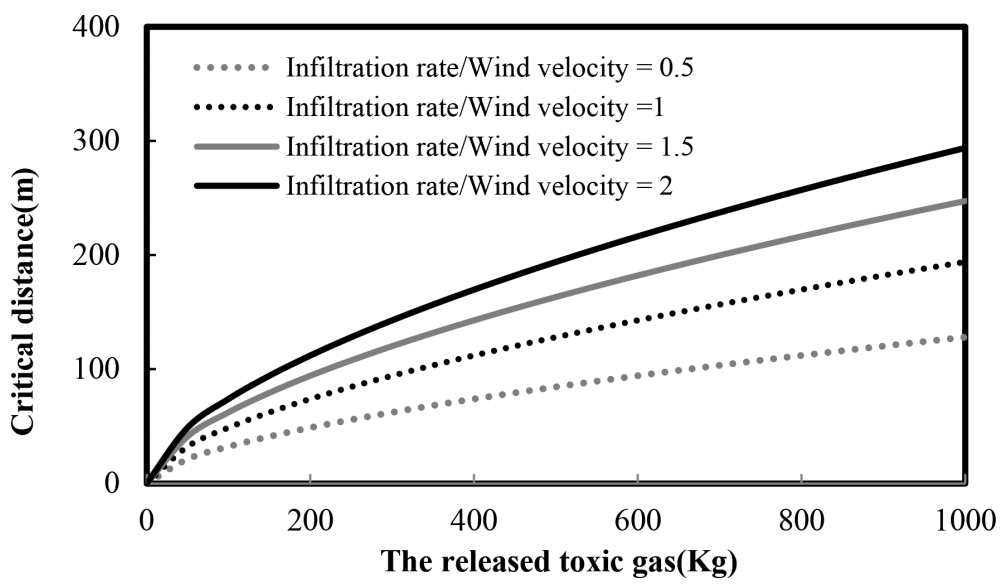

Figure 11: Critical distances for atmospheric stability class A with total released toxic gas. $(\mathrm{C}=20 \mathrm{ppm})$.

For $\bar{\eta} / U_{W}=2$ and atmospheric stability class $=\mathrm{F}$,

$$
X_{2-c r} \cong 270.46 Q^{\frac{1}{1.5}}
$$

The eqns (3) and (4) may be used to set the minimum separation distance of the chlorine gas cylinder from residential area and from vulnerable building, respectively.

If an accident of chlorine gas is happened for the case of $\bar{\eta} / U_{W}=0.003$ and atmospheric stability class $=\mathrm{A}$, person inner the distance will have always life-threatening. For other case, the consequences will depend on atmospheric conditions. 


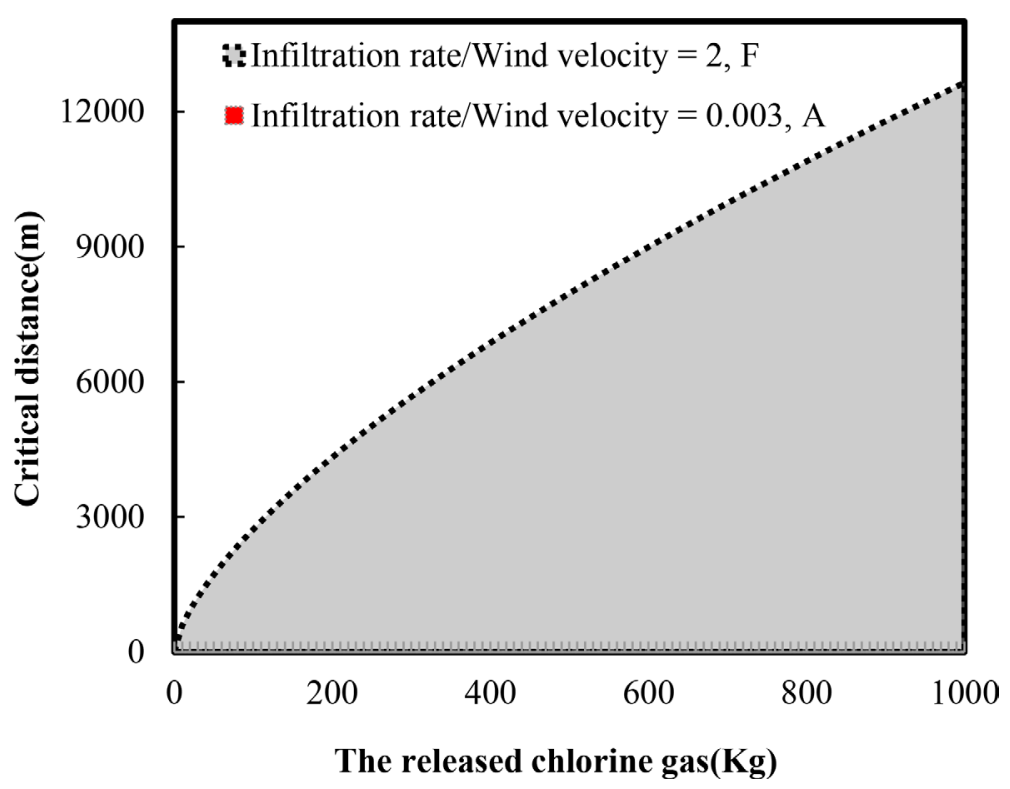

Figure 12: Critical distances for the worst scenario and the other case.
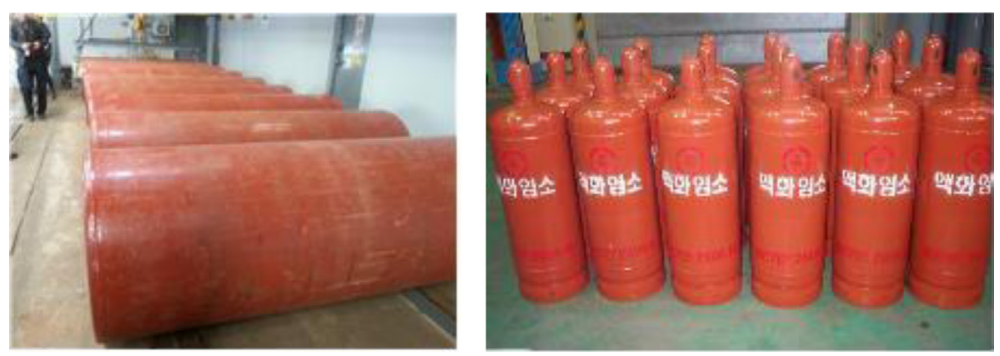

Figure 13: Type of chlorine gas cylinder (1 Tone/47 L).

\section{CONTROL THE SAFETY OF CHLORINE GAS CYLINDER}

There are two kinds of chlorine gas cylinder as shown in Fig. 12. The small one is a $150 \mathrm{lb}$ cylinder and the large one is a 1 ton cylinder. The safety distances associated with the catastrophic failure of the chlorine cylinder can be estimated by using eqns (3) and (4). The distances for worst case are 2,108 $\mathrm{m}$ and $12,653 \mathrm{~m}$, and the other case is $1.27 \mathrm{~m}$ and $6.36 \mathrm{~m}$, respectively. When the chlorine cylinder of 1 ton is delivered to end user where the location is closer to vulnerable building less than $6.36 \mathrm{~m}$, the tracking system automatically notify to the safety manager that the 1 tone of chlorine cylinder have to be replaced by several $150 \mathrm{lb}$ cylinders to improve the safety. If there are buildings in the distance of 12, $653 \mathrm{~m}$ for 1 ton or $2,108 \mathrm{~m}$ for $150 \mathrm{lb}$ from the delivery point, the buildings could not be used as a shelter protecting person against catastrophic failure of the cylinder. These cases should be addressed by means to reduce the frequency and/or consequences to an acceptable level. The consequences 
can be reduced by using a secondary enclosure system with scrubber, and water deluge of soluble chlorine gas. The failure frequency can be reduced by checking or/and inspecting the cylinder frequently.

If the toxic gas cylinder is not delivered to the end user during a certain time, there is a possibility that the cylinder may be misused such as a terror. Therefore, the tracking system checks the time of delivery and automatically notifies to the safety manager if it is over the certain time duration.

\section{CONCLUSIONS}

The tracking system is fully operational throughout South Korea. The distance toxic effecting on a person in a building in down-wind ranges from under ten meters for extremely atmospheric unstable and is up to over thousand meters for extremely atmospheric stable. The unique toxic gas cylinder tracking system can not only use as a safety management tool to prevent terror or to reduce the consequences of the accident but also has benefit in the gas company as to keep the balance of gas cylinder, to prevent lost or stolen of cylinder, and to identify the returned cylinder.

\section{REFERENCES}

[1] Song, B.H. \& Jo, Y.D., Safety management system for emergency handling of environmental toxic gas release. Korean Journal of Hazardous Materials, 2(2), pp. 26-30, 2014.

[2] https://en.wikipedia.org/wiki/Radio-frequency_identification

[3] Jo, Y.D. \& Park, K.D., Emergency response plane of toxic gas release with considering ventilation ratio and meteorological conditions, 2016.

[4] Shepard, S., A history of bar codes. In RFID: Radio Frequency Identification, McGraw Hill: New York, pp. 12-26, 2005

[5] Gas cylinders monitoring by wireless tags, United States Patent No. US 7,619,523 B2, 2009.

[6] Automated gas cylinder tracking system, United States Patent No. 5,953,682, 2009. 